\title{
Looking to the future of drug-induced liver injury: an interview with Paul B Watkins
}

Paul B Watkins*,1

${ }^{1}$ Director of the University of North Carolina Institute for Drug Safety Sciences, Howard Q Ferguson Distinguished Professor of Pharmacy, Eshelman School of Pharmacy, University of North Carolina at Chapel Hill, NC, USA

* Author for correspondence: pbwatkins@med.unc.edu

Paul Watkins talks to Francesca Lake, Head of Open Access Publishing: Paul is The Howard Q Ferguson Professor in the Eshelman School of Pharmacy at The University of North Carolina at Chapel Hill (UNC, NC, USA), and an expert in drug safety and drug-induced liver injury (DILI). Since its formation in 2009, he has been the director of the University of North Carolina Institute for Drug Safety Science. He is also Director of the DILI-sim Initiative and Chairman of the Board of Directors for the spin-off company, DILIsym Services, Inc. He is chair of the steering and genetics committees for the US Drug-Induced Liver Injury Network supported by the NIH and has been the recipient of a number of awards.

First draft submitted: 13 March 2017; Accepted for publication: 22 March 2017; Published online: 11 September 2017

Keywords: computer modeling • drug-induced liver injury • drug safety • toxicology

Can you tell us a little about your background \& what led you into hepatology, \& drug-induced liver injury specifically?

I am a physician-scientist. After medical school, I did an internship in residency in internal medicine, and then decided to do a fellowship in gastroenterology. During the course of the fellowship, I did research for the first time and I got hooked. The research I was doing involved how the liver handles drugs. I realized at that point that I wanted a career in academic medicine with a really strong research component. At the time, concentrating on liver disease was the easiest route for that; this was around the 1980s, when liver disease was fascinating but there was not an extensive clinical load there. Overall, that would allow me to be a good doctor for liver patients but also spend the majority of my time doing research. I should mention that I was wrong about this, it was not long until the clinical load grew exponentially due to the advent of liver transplantation and treatments of viral hepatitis.

\section{What research are you working on at the moment?}

I am currently trying to find out why good drugs are sometimes bad for the liver. In other words, how can a drug that is safe for $99.99 \%$ of patients create a life-threatening liver injury resulting in death or transplant in a very rare patient? What is it about that drug, and what is it about that patient? That is my area of research both in the laboratory but also clinically.

\section{Have you made any strides in this area?}

I think so. It appears that most rare or sudden liver injuries due to drugs are actually an immune attack on the liver. It seems like your body believes the liver is infected with a virus and attacks to kill what it thinks are virus-infected cells. However, there is no virus. Somehow the drug, in a very rare subgroup of patients, creates stress in the liver cells and somehow fools the body into thinking it needs to kill liver cells. Of course, many others have contributed to this progress in understanding drug-induced liver injury (DILI) .

I currently chair the Steering and Genetics committee for the US Drug-Induced Liver Injury Network, which is now entering its 15 th year. We have gone around the country and found people who have had these very rare reactions and created a registry and tissue bank so we and others can study them. I also do research in the laboratory that involves culturing liver cells and mouse models, and have been putting all this together to best address this 
problem. Where we really piece this all together is in the modeling effort called the Drug-Induced Liver Injury-sim (DILI-sim) Initiative.

\section{Can you tell us about the DILI-sim Initiative, and your work with it?}

The DILI-sim initiative was actually started as what is called a CRADA with the US FDA. The FDA has been from the get-go a strong supporter of the idea that you could model with a computer what was going on in the liver, and this approach could be utilized to both understand and predict DILI, ultimately making drugs safer. The DILI-sim Initiative, which is now in its 6th year, has had 16 of the top 20 pharmaceutical companies as partners; that is, they have provided resources and scientific expertise, and in some cases performed research to fill in the identified gaps in knowledge. What this initiative does is try to break down the actual mechanisms or processes whereby a drug can hurt the liver and express these in mathematical equations. DILI can be 'solved' essentially by these computer programs. The product is software called DILIsym ${ }^{\circledR}$, and this is a registered trademark for version control - we are now on version $5 \mathrm{~b}$. To use the software, you take your drug and run it through some commercially available tests, and you take the data from those tests and plug it into the software. It will then predict whether your drug is safe in the average person, and also whether it is safe in a simulated patient population that has been created by varying the model parameters to simulate the genetic and environmental factors that make patients different. This approach can explain why you can see a severe liver injury in one patient when the drug is safe for the vast majority of patients.

\section{How accurate is that software?}

The software has been built with a series of test drugs, many of which have been provided by our partners and looked safe in animals but produced DILI in people. We have now moved into the validation stage of the software, and to date approximately 30 different drugs have been used to test the model's accuracy. So far for more than $80 \%$ of the drugs tested, the software has accurately predicted liver safety liability that in reality was only found by giving the drug to people. The evaluation of the model is pretty rigorous - it is not just to get a yes or no regarding safety of a drug but a series of parameters that are used to judge the success of the model - the frequency of observed toxicity, the latency in onset of the injury relative to starting drug treatment, and so on. About $80 \%$ of the test compounds so far have been judged as having good or excellent prediction in the model, which was a big surprise to me. Essentially, there are just three mechanisms in the model that are very good at predicting liver liabilities in new drug candidates.

\section{How far are you away from being able to use the model in early-stage clinical drug development?}

The problem right now is that it takes $2-3$ months to gather the right data and plug it into the model. This is simply too long to be useful for early preclinical decisions. So far, the model has mainly been applied once the drug has gone into people and unexpected liver safety signals emerge. The model has in fact been part of some regulatory submissions, including a recent new drug application (NDA) and a presentation for an FDA advisory committee. The model has been found to be useful in explaining, predicting and avoiding liver injury once the drug has gone into people, or for selecting backup compounds when the lead candidate fails due to liver toxicity. We are working to make modifications in the process that will improve its efficiency and make application in preclinical screening feasible.

\section{You have been awarded numerous awards for your work. What would you say has been your greatest achievement?}

Putting together and directing the DILI-sim initiative is definitely my greatest achievement. I am very proud of the DILI-sim initiative - putting the aggregate understanding of DILI together in a useful tool that is already helping to speed up drug development and make it more efficient, and overall improving the safety of drugs. That is definitely my greatest career achievement, no question.

\section{What challenges are we still facing in terms of accurately predicting liver toxicity for new drugs?}

The challenges are really improving the speed and efficiency of this modeling process so that it can be used right at the earliest compound-screening stage. As I noted, we are working in that direction, but currently the process 
takes too much time. I have no doubt we will get there. The other issue is that the model does not have all the components of immunity that are clearly often important, particularly in the very rare episodes of DILI that occur after weeks or months of treatment. We have mounted a major effort to build these immune mechanisms into the model and have identified the appropriate academic and industry scientists to help us with this effort.

\section{How many iterations of the software do you think you might need to get there?}

The DILI-sim initiative is run in 3-year blocks; we get 3 years of commitment from our partners. 2017 is the final year of the second 3-year period. We are confident we will be supported by our partners for the next 3-year iteration, where we will be making the modeling process more efficient so it can be done earlier in development. To do that, we will be looking for metabolically competent cell culture systems that can rapidly identify what the relevant DILI mechanisms are and account for the effects of metabolites as well as the parent compound. In addition, in the next 3 years we will be adding in adaptive immunity components to the model. At the end of the next 3 years, I am hopeful we will have an optimally useful tool that will have a major public health impact.

\section{With increasing research looking to in vitro or in silico methods, what do you personally think the future of drug toxicity assessment looks like?}

I think there will be a combination of the two. The field is moving away from animal testing, although this testing will not leave us any time soon. I see us moving toward the so-called 'human-on-a-chip' but I also see in silico modeling, what is called quantitative systems pharmacology or quantitative systems toxicology, exploding over the next decade. I think it will be extremely exciting as it will allow much smaller clinical trials in people to establish efficacy and safety than is currently the case. This means delivering important new drugs sooner to the patients that need them, and at a cheaper price.

\section{If you had unlimited funding, what research would you perform, \& why?}

I would greatly expand our own work in what is called organotypic culture, to really develop a human liver culture system that would allow us to rapidly identify what the relevant toxicity pathways are for each new drug candidate, so we can focus the data collection and modeling effort. This would include the role of metabolites and would also allow us to rapidly predict the relationship between concentrations of the drug outside and inside the liver cell to better estimate what the liver exposure would be in patients. Ideally, we would be able to get measurements from that culture system that could be plugged directly into DILI-sim. That would be ideal!

Financial \& competing interests disclosure

Dr. PB Watkins is chairman of the Scientific Advisory Board for the DILI-sim Intiative. He also has a financial interest in Simulations Plus that acquired DILIsym Services, Inc which was the spin off company from the DILI-sim Initiative. The interviewee has no other relevant affiliations or financial involvement with any organization or entity with a financial interest in or financial conflict with the subject matter or materials discussed in the manuscript apart from those disclosed.

No writing assistance was utilized in the production of this manuscript.

Open access

This work is licensed under the Creative Commons Attribution 4.0 License. To view a copy of this license, visit http:// creativecommons.org/licenses/by/4.0/ 
(

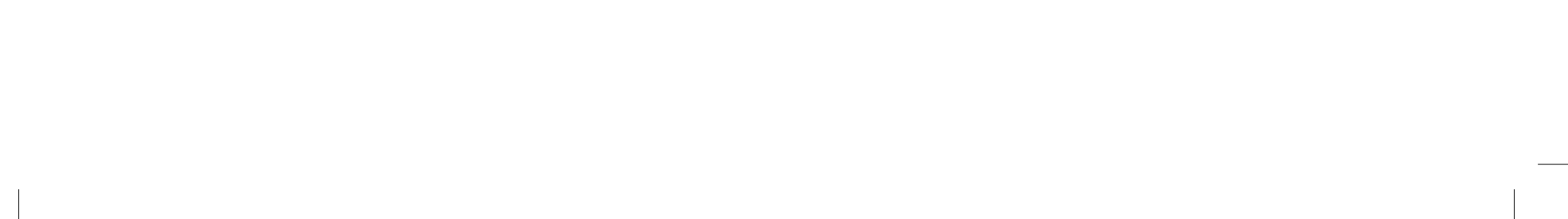

